\title{
28 Research Soure \\ LncRNA NRON Inhibits Osteosarcoma Cell Proliferation, Invasion by Regulating MVB12B
}

\section{Yancheng Liu}

Tianjing Hospital

Jingyu Zhang

Tianjing Hospital

\section{Yongqiang Jiao}

Tianjing Hospital

\section{Shuwei Ma}

Tianjing Hospital

\section{Xinlong Ma}

Tianjing Hospital

Yongcheng Hu ( $\nabla$ yongchenghutj@aliyun.com )

Department of Bone and Soft Tissue Tumors, Tianjing Hospital https://orcid.org/0000-0002-82224133

\section{Research article}

Keywords: IncRNA, osteosarcoma, NRON, Rescue

Posted Date: September 4th, 2020

DOI: https://doi.org/10.21203/rs.3.rs-69251/v1

License: (a) (i) This work is licensed under a Creative Commons Attribution 4.0 International License.

Read Full License 


\section{Abstract}

Aims: Long noncoding RNA have been proved as important regulator in various diseases. NRON was a newly identified tumor-related IncRNA, and previous studies have reported its function in hepatocellular carcinoma and heart failure. However, the function and mechanism of IncRNA NRON in osteosarcoma still unknown.

Methods: Cell proliferation, invasion, migration and apoptosis were detected via CCK-8, transwell assay and Western. Bioinformatics analysis was used to predict the potential target of NRON. Rescue experiment was performed to identify the relationship between NRON and MVB12B.

Results: The expression of IncRNA NRON was significantly downregulate in osteosarcoma tissues and cell lines. Knockdown of NRON promoted cell proliferation, invasion and EMT. Overexpression of NRON inhibited cell proliferation, invasion and EMT. Bioinformatics analysis predicted that MVB12B was the direct target. The expression of MVB12B was significantly upregulated in osteosarcoma tissues and cell lines. Rescue experiment further confirmed the relationship between NRON and MVB12B. Overexpression of MVB12B completely reversed the function of NRON.

Conclusion: Taken together, our results comprehensively analyzed the function of NRON in osteosarcoma and provided possible mechanism that NRON inhibited osteosarcoma development by regulating MVB12B. Thus, our study may offer a potential therapeutic target for treating osteosarcoma.

\section{Introduction}

Osteosarcoma is the most prevalent primary malignant bone cancer and is most common in children and teenagers[1]. It is most common that people often die due to distant metastasis[2]. The pathological development of osteosarcoma involved various process, including invasion[3], EMT[4] and angiogenesis[5]. Despite remarkable advancement in the biomarkers and treatment of osteosarcoma, there still numerous questions waiting for answered[6]. Thus, exploring new targets and effective drugs are clearly warranted.

Long noncoding RNA is a kind of noncoding RNA that longer than $200 \mathrm{nt}$ with limited coding potential[7]. Accumulating studies have reported the various role of IncRNA in different biological and pathological process $[8,9]$. For example, IncRNA PANDAR dictates the chemoresistance of ovarian cancer via regulating SFRS2-mediated p53 phosphorylation[10]. Over-expressed IncRNA HOTAIRM1 promotes tumor growth and invasion through up-regulating HOXA1 in glioblastoma multiforme[11]. LncRNA XIST mediates bovine mammary epithelial cell inflammatory response via NF-KB/NLRP3 inflammasome pathway[12]. Importantly, increasing evidences have demonstrated that dysregulated expression of IncRNA will influence the development of various human cancers $[13,14]$. There is no doubt that exploring the function and mechanism of IncRNAs will provide new choice for drug administration[15]. 
Previous studies have revealed the function of IncRNA NRON, a newly identified tumor-related IncRNA, in hepatocellular carcinoma and heart failure[16]. However, the function and mechanism of IncRNA NRON in osteosarcoma still unknown. In this study, we found that knockdown of NRON promoted cell proliferation, invasion and EMT. Bioinformatics analysis predicted that MVB12B is the potential target. The expression of MVB12B was significantly upregulated in osteosarcoma tissues and cell lines. Rescue experiment further confirmed the relationship between NRON and MVB12B. Overexpression of MVB12B completely reversed the function of NRON. Taken together, our results comprehensively analyzed the function of NRON in osteosarcoma and provided possible mechanism that NRON inhibited osteosarcoma development by regulating MVB12B.

To be brief, by conducting these comprehensive experiments, we want to explore the inner association between IncRNA NRON and MVB12B and demonstrate that IncRNA NRON could influence the progress of osteosarcoma by regulating MVB12B. The current research may explore the new target for inhibiting osteosarcoma.

\section{Materials And Methods}

\section{Cell culture}

The cell lines, KHOS, U2OS, HOS, Saos-2, MG63 and HFOB1.19 used in this study were purchased from American Type Culture Collection (ATCC) or maintained in our lab. U2OS and HOS cells were cultured in DMEM medium (Gibco, USA) containing $10 \% \mathrm{FBS}$ (Gibco, USA). Cells were maintained at $37^{\circ} \mathrm{C}$ in $5 \% \mathrm{CO}_{2}$ incubator.

\section{Cell transfection}

siRNA-NRON, negative control, overexpression plasmid and NC plasmid were purchased from Gene Pharma (Shanghai, China). Briefly, Cell lines U2OS and HOS were transfected with different vector with lipo3000 reagent (Invitrogen) following the manufacturer's protocol when cell confluence reached $40 \%$. Cells were cultured for $72 \mathrm{~h}$ before harvesting for further research.

\section{Cell proliferation}

HOS and U2OS cells were transfected with si-NC, si-NRON, NC, NRON, MVB12B and NC as mentioned above. Cells were seeded (2000 cells per well) into 96-well plates. After $48 \mathrm{~h}$ transfection, $10 \mu \mathrm{L} \mathrm{CCK}-8$ reagent was added to each well at $0 \mathrm{~h}, 24 \mathrm{~h}, 48 \mathrm{~h}, 72 \mathrm{~h}$ and then the optical density was measured at 450 $\mathrm{nm}$ after $2 \mathrm{~h}$ incubation.

\section{Transwell assay}

U2OS and HOS cells were re-suspended in $100 \mu \mathrm{L}$ serum-free medium and were plated in the top chamber of each insert (8- $\mu \mathrm{m}$ pore size, Corning, USA) with a Matrigel-coated membrane (BD Bioscience, San Jose, USA) for the transwell assay. Lower chambers of the inserts were filled with DMEM medium with $10 \%$ 
FBS. Twenty-four hours later, cells invaded to the lower surface of the insert were fixed, stained with crystal violet and counted under a light microscope.

\section{Real-time PCR}

Total RNA was extracted and lysed by TRIzol reagent (Thermo Fisher, USA). RNA reverse transcription was performed using a PrimeScript ${ }^{\mathrm{TM}} \mathrm{RT}$ reagent Kit with gDNA eraser (Takara, Japan) according to the manufacturer's instructions, and cDNA was performed by qRT-PCR using SYBRß Premix Ex Taq ${ }^{\text {TM }}$ (Takara, Japan). The data were normalized using $\beta$-actin levels and further analyzed by the $2-\triangle \triangle C T$ method.

\section{Western blotting}

Proteins from HOS and U2OS cells were extracted by using the RIPA buffer. Total protein was quantified using BCA protein assay kit (Pierce, USA). Protein samples were resolved by $10 \%$ SDS-PAGE gel ( $80 \mathrm{~V}$, $120 \mathrm{mins}$ ) and transferred to polyvinylidene difluoride (PVDF) membrane (300mA, 90 mins). And membranes were blocked with $5 \%$ pure milk for 1 hour. Then they were incubated with primary antibodies against E-cadherin (1:1000, CST, USA), N-cadherin (1:1000, CST, USA), Vimentin (1:1000, CST, USA), MVB12B(1:1000, Santa Cruz, CA), $\beta$-actin $\left(1: 5000, C S T\right.$, USA) at $4{ }^{\circ} \mathrm{C}$ overnight, followed by incubation with HRP conjugated secondary antibodies (Abcam, CA, USA) for $1 \mathrm{~h}$ at room temperature. The bands were visualized by using the ECL kit (Thermo Fisher Scientific, Rockford, USA).

\section{Statistical analysis}

We used SPSS 21.0 to calculate the values (means \pm standard error of the mean (SEM). And statistical analyses were analyzed using two-sided Student's t-test or one-way ANOVA. The statistical significance was $\mathrm{P}<0.05$.

\section{Results}

\section{Biological features of IncRNA NRON}

To study the function of IncRNA NRON, we firstly analyzed the biological features of IncRNA NRON in osteosarcoma. Our results showed that the expression of IncRNA NRON was significantly downregulated in osteosarcoma tissues (Fig.1A). Similar, we also analyzed the expression of IncRNA NRON in osteosarcoma cell lines (Fig.1B). Real-time PCR results showed that the expression of IncRNA NRON was significantly downregulated in osteosarcoma cell lines. In addition, we analyzed the expression of IncRNA NRON in different mouse organs. Our results showed that the expression of IncRNA NRON was highly in muscle tissues, and expressed in various tissues, suggesting that IncRNA NRON may also possess function in other disease (Fig.1C). Lastly, we analyzed the conservation level of IncRNA NRON in different animals. Bioinformatics analysis showed that IncRNA NRON is highly conserved in mouse, dog, elephant and chicken (Fig.1D), suggesting IncRNA NRON may play an important role in the biological process.

\section{Knockdown of IncRNA NRON promoted cell proliferation and invasion}


To study the function of IncRNA NRON, we constructed knockdown siRNA. The knockdown efficiency was confirmed via real-time PCR. The expression of IncRNA NRON was significantly reduced in HOS and U2OS cell lines (Fig.2A). We performed CCK-8, transwell and western blot to evaluate the proliferation, invasion and EMT. CCK-8 results showed that knockdown of IncRNA NRON promoted cell proliferation in HOS and U2OS cell lines (Fig.2B). Transwell assay showed that downregulated of IncRNA NRON increased the cell invasion number (Fig.2C). Lastly, we examined the EMT markers, E-cadherin, N-cadherin and Vimentin, to evaluate the influence of IncRNA NRON. Our results showed that knockdown of IncRNA NRON reduced the protein level of E-cadherin, while increased the protein level of $\mathrm{N}$-cadherin and Vimentin in HOS and U2OS cell lines (Fig.2D). Thus, we proved that knockdown of IncRNA NRON can promote the development of osteosarcoma.

\section{Overexpression of IncRNA NRON inhibited cell proliferation and invasion}

To further study the function of IncRNA NRON, we constructed overexpression vector. The overexpression efficiency was verified via real-time PCR. The expression of IncRNA NRON was significantly increased in HOS and U2OS cells (Fig.3A). We performed CCK-8, transwell and western blot to evaluate the proliferation, invasion and EMT. CCK-8 results showed that overexpression of IncRNA NRON inhibited cell proliferation in HOS and U2OS cell lines (Fig.3B). Transwell assay showed that upregulated of IncRNA NRON reduced the cell invasion number (Fig.3C). Lastly, we examined the EMT markers, E-cadherin, Ncadherin and Vimentin, to evaluate the influence of IncRNA NRON. Our results showed that overexpression of IncRNA NRON increased the protein level of E-cadherin, while decreased the protein level of N-cadherin and Vimentin in HOS and U2OS cell lines (Fig.3D). Thus, we proved that overexpression of IncRNA NRON can inhibit the development of osteosarcoma.

\section{Biological features of MVB12B}

To explore the mechanism of IncRNA NRON involved in osteosarcoma, we used bioinformatics analysis to predict the potential target of IncRNA NRON. As shown in Fig.4A, MVB12B is the neighboring gene of IncRNA NRON. The expression of MVB12B in different tissues was showed in Fig.4A, suggesting that MVB12B may possess function in the tumor development. Next, we analyzed the biological features of MVB12B in osteosarcoma. The expression of MVB12B was significantly increased in osteosarcoma tissues (Fig.4B). We also analyzed the expression of MVB12B in cancer cell lines, which is upregulated in osteosarcoma cell lines (Fig.4C). We wondered the relationship between IncRNA NRON and MVB12B. Overexpression of NRON reduced the expression of MVB12B and vice versa (Fig.4D). Similar results can be observed by western blot, suggesting that IncRNA NRON can negatively regulate the expression of MVB12B at mRNA and protein level (Fig.4E). Next, we performed experiments to assess the function of MVB12B in osteosarcoma. Firstly, we constructed overexpression vector of MVB12B. The expression of MVB12B was significantly upregulated in HOS and U2OS cells (Fig.4F). CCK-8 assay showed that overexpression of MVB12B significantly promoted cell proliferation in HOS and U2OS cells, as indicated by CCK-8 assay (Fig.4G). Thus, our results showed that MVB12B is the potential target of IncRNA NRON.

\section{Reintroduction of MVB12B rescued the function of IncRNA NRON}


To verify the relationship between IncRNA NRON and MVB12B, we performed comprehensive rescue experiment to examine whether IncRNA NRON inhibit the osteosarcoma development by regulating MVB12B. Co-transfection group of IncRNA NRON and MVB12B significantly increased the cell invasion number compared with IncRNA NRON overexpression group in HOS and U2OS cells (Fig.5A). CCK-8 assay showed that reintroduction of MVB12B completely blocked the inhibit effect induced by overexpression of IncRNA NRON (Fig.5B). Western blot showed that reintroduction of MVB12B reversed the effect of IncRNA NRON, as indicated by decreased protein level of E-cadherin and increased protein level of Ncadherin and Vimentin (Fig.5C, Fig.5D). Thus, our results showed that IncRNA NRON can inhibit cell proliferation and invasion by negatively regulating MVB12B.

\section{Discussion}

Previous studies have revealed the function of IncRNA NRON, a newly identified tumor-related IncRNA, in hepatocellular carcinoma and heart failure. However, the function and mechanism of IncRNA NRON in osteosarcoma still unknown. In this study, we found that knockdown of NRON promoted cell proliferation, invasion and EMT. Bioinformatics analysis predicted that MVB12B is the direct target. The expression of MVB12B was significantly upregulated in osteosarcoma tissues and cell lines. Rescue experiment further confirmed the relationship between NRON and MVB12B. Overexpression of MVB12B completely reversed the function of NRON. Taken together, our results comprehensively analyzed the function of NRON in osteosarcoma and provided possible mechanism that NRON inhibited osteosarcoma development by regulating MVB12B.

Long noncoding RNA has been showed to play important roles in various disease[17]. For example, Long noncoding RNA LINC00511 contributes to breast cancer tumourigenesis and stemness by inducing the miR-185-3p/E2F1/Nanog axis[18]. LncRNA UCA1 promotes tumor metastasis by inducing miR-203/ZEB2 axis in gastric cancer[19]. IncRNA NRON has been reported in numerous diseases, which is highly expressed in resting CD4(+) T lymphocytes, could be involved in HIV-1 latency by specifically inducing Tat protein degradation[20]. NRON IncRNA potently suppresses the viral transcription by decreasing the cellular abundance of viral transactivator protein Tat. However, little is known about the function of NRON in osteosarcoma. In our study, we utilized overexpression and knockdown vector of NRON in osteosarcoma cell lines to study the invasion, proliferation and EMT indexes. Our results showed that NRON possessed an anti-tumor function, as indicated by inhibited proliferation and cell invasion number.

The mechanism of IncRNA can be diverse, including regulation of gene transcription, post-translational regulation, epigenetic regulation[21]. In post-translational regulation, for example, IncRNA RMST Suppressed GBM Cell Mitophagy through Enhancing FUS SUMOylation[22]. Lnc-CCDST promotes DHX9 degradation by serving as a scaffold to facilitate the formation of MDM2 and DHX9 complexes[23]. In epigenetic regulation, SWINGN influences the ability of the SWI/SNF complexes to drive epigenetic activation of specific promoters, suggesting a SWI/SNF-RNA cooperation to achieve optimal transcriptional activation[24]. Previous studies have shown that LncRNA NRON alleviates atrial fibrosis via promoting NFATc3 phosphorylation[16]. Long noncoding RNA NRON contributes to HIV-1 latency by 
specifically inducing tat protein degradation[20]. NRON can function through epigenetic regulation and post-translational regulation. In our study, we found the neighboring gene MVB12B was significantly changed upon IncRNA NRON treatment. Overexpression of IncRNA reduced the expression of MVB12B at mRNA and protein level. Thus, we speculate that NRON may influence the level of MVB12B at transcription level. Further rescue experiment revealed that re-introduction of MVB12B can completely restored the function of IncRNA NRON.

NRON has been reported that directly bind with Tat to the ubiquitin/proteasome component to facilitate Tat protein degradation[20]. There still another report about NRON in atrial fibrosis, which can inhibit fibroblast proliferation and downstream targets, including collagen I and collagen III, through promoting NFATc3 phosphorylation. To date, our results was the first study of function and mechanism of NRON in osteosarcoma. Interestingly, the mechanism of NRON in osteosarcoma was different with HIV infection and atrial fibrosis, suggesting that NRON may associated with numerous mechanisms and involved in different pathological process. Thus, further study of NRON in other disease is clearly warranted.

To sum up, our study uncovered the role of IncRNA NRON in osteosarcoma, which possessed an antitumor role in the development of osteosarcoma. Further studies of IncRNA undoubtedly deepen understanding of the occur and pathology of osteosarcoma. Thus, our study may offer a potential therapeutic target for treating osteosarcoma.

\section{Declarations}

\section{Acknowledgments}

This work was supported by National Natural Science Foundation (81472140).

Conflict of Interest: The authors declare no competing financial interests.

\section{Data availability:}

The data used to support the findings of this study are included within the article.

\section{Author contribution:}

Xinlong Ma, Yongcheng Hu designed the research. Yancheng Liu, Jingyu Zhang, Yongqiang Jiao performed the experiments and wrote the manuscript. Yongqiang Jiao and Shuwei Ma analyzed the data.

\section{References}

1. Sun X, Yang L, Geng X, Wang R, Zhang Z. Increased expression of IncRNA HULC indicates a poor prognosis and promotes cell metastasis in osteosarcoma. International Journal of Clinical Experimental Pathology. 2015;8(3):2994-3000. 
2. Peng ZQ, Lu RB, Xiao DM, Xiao ZM, Increased expression of the IncRNA BANCR and its prognostic significance in human osteosarcoma. Genetics \& Molecular Research, 2016. 15(1).

3. Sun R, Muheremu A, Hu Y. miRNA-30c can be used as a target in the diagnosis and treatment of osteosarcoma. Onco Targets Ther. 2018;11:9091-9.

4. Gao G, Tian Z, Zhu HY, Ouyang XY. miRNA-133b targets FGFR1 and presents multiple tumor suppressor activities in osteosarcoma. Cancer Cell Int. 2018;18:210.

5. Dai Z, Tang H, Pan Y, Chen J, Li Y, Zhu J. Gene expression profiles and pathway enrichment analysis of human osteosarcoma cells exposed to sorafenib. FEBS Open Bio. 2018;8(5):860-7.

6. An R, Schmid R, Klausing A, Robering JW, Weber M, Bäuerle T, Detsch R, Boccaccini AR, Horch RE, Boos AM, Weigand A, Proangiogenic effects of tumor cells on endothelial progenitor cells vary with tumor type in an in vitro and in vivo rat model. Faseb j., 2018: p. fj201800135RR.

7. X W, D C, F Z. L. M, and W. Q, Long noncoding RNA TUSC7 inhibits cell proliferation, migration and invasion by regulating SOCS4 (SOCS5) expression through targeting miR-616 in endometrial carcinoma. Life sciences. 2019;231:116549.

8. Kong Y, Chih-Heng H, Alonso LC. ANRIL: A IncRNA at the CDKN2A/B Locus With Roles in Cancer and Metabolic Disease. Frontiers in Endocrinology. 2018;9:405-

9. Gai HY, Wu C, Zhang Y, Wang D. Long non-coding RNA CHRF modulates the progression of cerebral ischemia/reperfusion injury via miR-126/SOX6 signaling pathway. Biochem. Biophys. Res. Commun., 2019.

10. Wang H, Liu M, Fang L, Jiang J, Zhang Z, Kuang Y, Wang B, Shang X, Han P, Li Y, Li P. The cisplatininduced IncRNA PANDAR dictates the chemoresistance of ovarian cancer via regulating SFRS2mediated p53 phosphorylation. Cell Death Dis. 2018;9(11):1103.

11. Li Q, Dong C, Cui J, Wang Y, Hong X. Over-expressed IncRNA HOTAIRM1 promotes tumor growth and invasion through up-regulating HOXA1 and sequestering G9a/EZH2/Dnmts away from the HOXA1 gene in glioblastoma multiforme. J Exp Clin Cancer Res. 2018;37(1):265.

12. Ma M, Pei Y, Wang X, Feng J, Zhang Y, Gao MQ. LncRNA XIST mediates bovine mammary epithelial cell inflammatory response via NF-KB/NLRP3 inflammasome pathway. Cell Prolif., 2018: p. e12525.

13. C Z, H L, L X, Y. N, and Y C, LncRNA ELFN1-AS1 promotes esophageal cancer progression by upregulating GFPT1 via sponging miR-183-3p. Biological chemistry, 2019.

14. S L, X. Y YJ, F. Q, and KP Y. Long Noncoding RNA NEAT1 Promotes Proliferation and Invasion via Targeting miR-181a-5p in Non-Small Cell Lung Cancer. Oncology research. 2018;26(2):289-96.

15. S L, JY L, L L, Y Y, C C, X H, W WB,XR,L, P Y, Z SW, L, H L, YE NJ,TF,WJ,Z. and S. X, Divergent IncRNAs Regulate Gene Expression Lineage Differentiation in Pluripotent Cells Cell stem cell. 2016;18(5):63752.

16. Wang Y, Xu P, Zhang C, Feng J, Gong W, Ge S, Guo Z. LncRNA NRON alleviates atrial fibrosis via promoting NFATc3 phosphorylation. Mol. Cell. Biochem., 2019. 
17. Xue $M$, Shi $D, X u G$, Wang $W$. The long noncoding RNA linc00858 promotes progress of lung cancer through miR-3182/MMP2 axis. Artif Cells Nanomed Biotechnol. 2019;47(1):2091-7.

18. Lu G, Li Y, Ma Y, Lu J, Chen Y, Jiang Q, Qin Q, Zhao L, Huang Q, Luo Z, Huang S, Wei Z. Long noncoding RNA LINC00511 contributes to breast cancer tumourigenesis and stemness by inducing the miR-185-3p/E2F1/Nanog axis. J. Exp. Clin. Cancer Res., 2018. 37(1): p. 289.

19. Gong P, Qiao F, Wu H, Cui H, Li Y, Zheng Y, Zhou M, Fan H. LncRNA UCA1 promotes tumor metastasis by inducing miR-203/ZEB2 axis in gastric cancer. Cell Death Dis. 2018;9(12):1158.

20. Li J, Chen C, Ma X, Geng G, Liu B, Zhang Y, Zhang S, Zhong F, Liu C, Yin Y, Cai W, Zhang H. Long noncoding RNA NRON contributes to HIV-1 latency by specifically inducing tat protein degradation. Nat Commun. 2016;7:11730.

21. Hochberg-Laufer $\mathrm{H}$, Schwed-Gross A, Neugebauer KM, Shav-Tal Y, Uncoupling of nucleo-cytoplasmic RNA export and localization during stress. Nucleic Acids Res., 2019.

22. Liu C, Peng Z, Li P, Fu H, Feng J, Zhang Y, Liu T, Liu Y, Liu Q, Liu Q, Li D, Wu M. IncRNA RMST Suppressed GBM Cell Mitophagy through Enhancing FUS SUMOylation. Mol Ther Nucleic Acids. 2020;19:1198-208.

23. Ding X, Jia X, Wang C, Xu J, Gao SJ, Lu C, A DHX9-IncRNA-MDM2 interaction regulates cell invasion and angiogenesis of cervical cancer. Cell Death Differ., 2018.

24. Grossi E, Raimondi I, Goñi E, González J, Marchese FP, Chapaprieta V, Martín-Subero JI, Guo S, Huarte M. A IncRNA-SWI/SNF complex crosstalk controls transcriptional activation at specific promoter regions. Nat Commun. 2020;11(1):936.

\section{Figures}
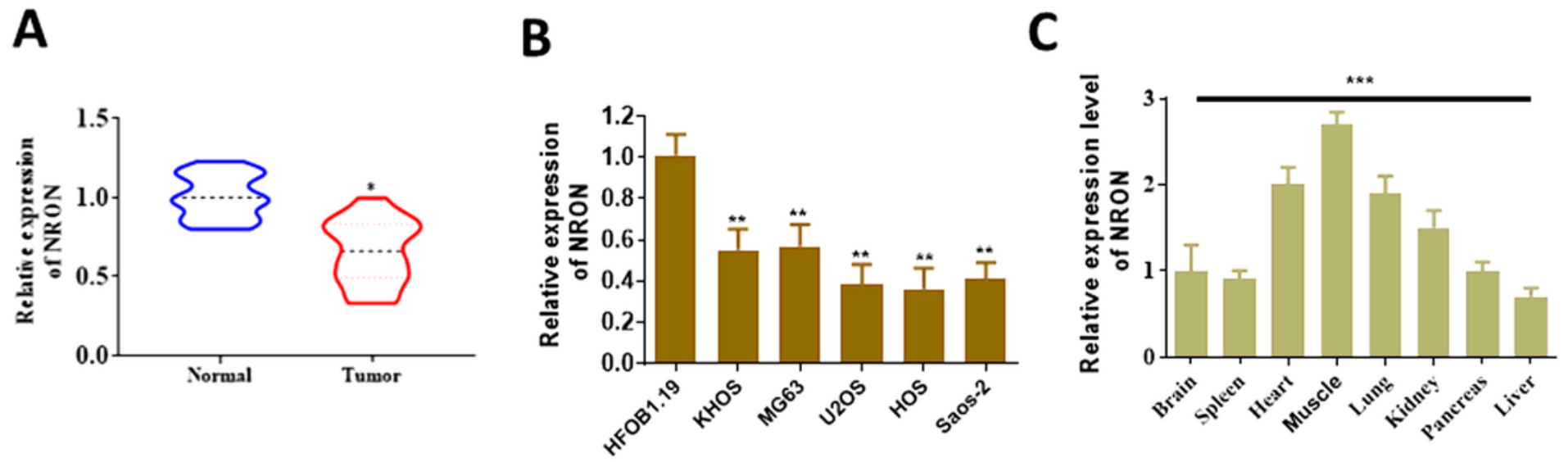

D

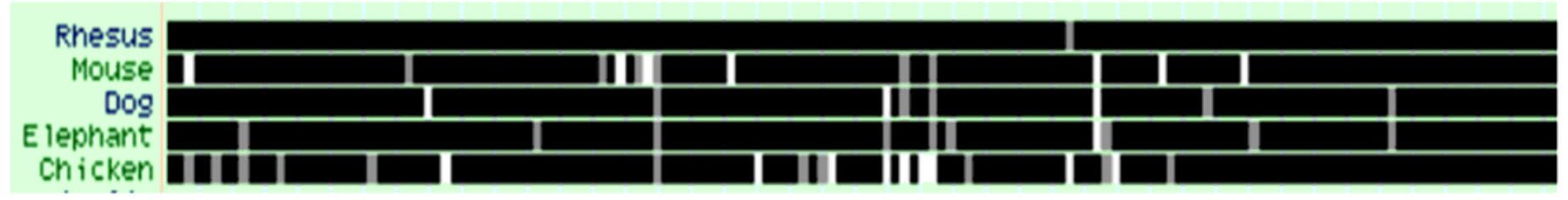


Figure 1

Biological features of IncRNA NRON. A. The expression of NRON was downregulated in tumor tissues. B. The expression of IncRNA NRON was downregulated in cell lines. C. Multi-tissue analysis revealed the expression of IncRNA NRON in different tissues. D. IncRNA NRON is highly conserved in different animals.

A

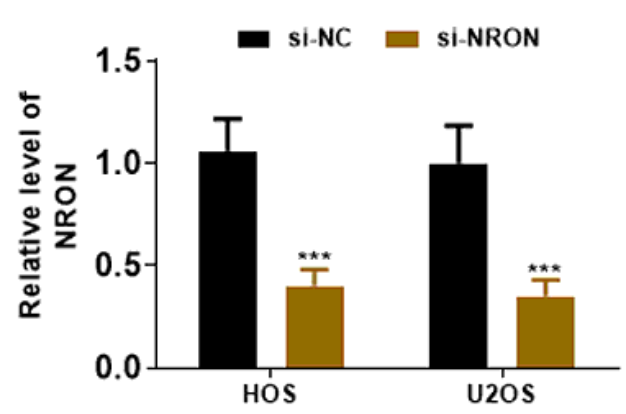

C

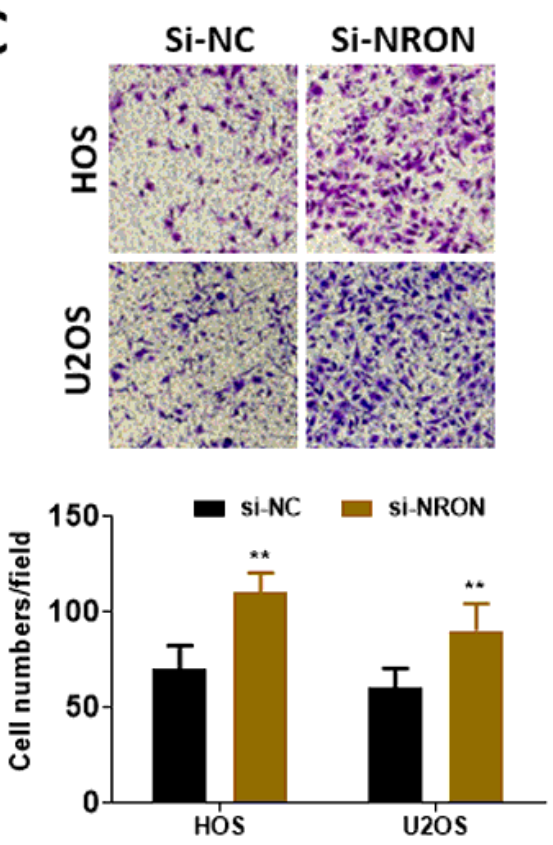

B
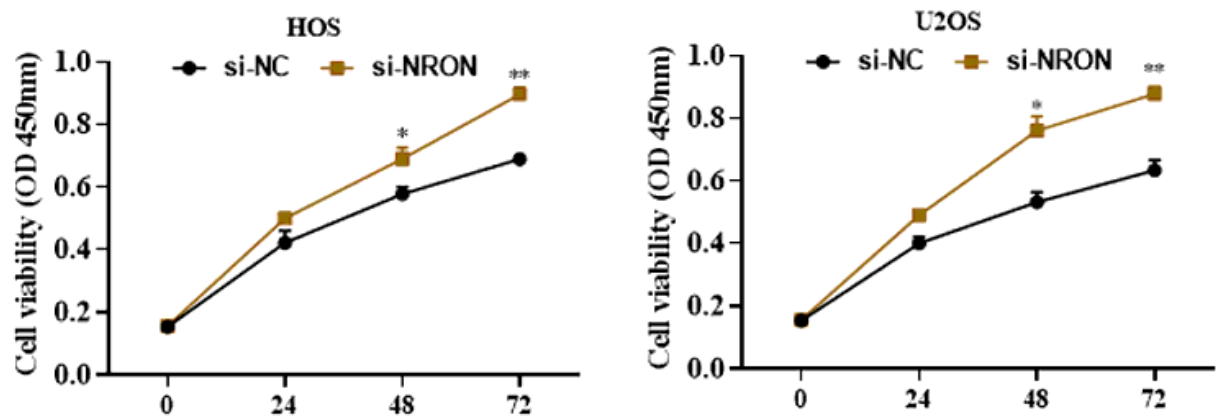

D

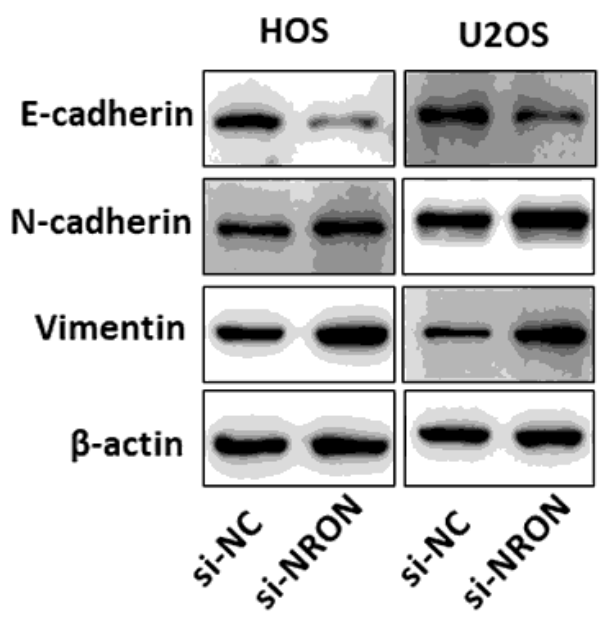

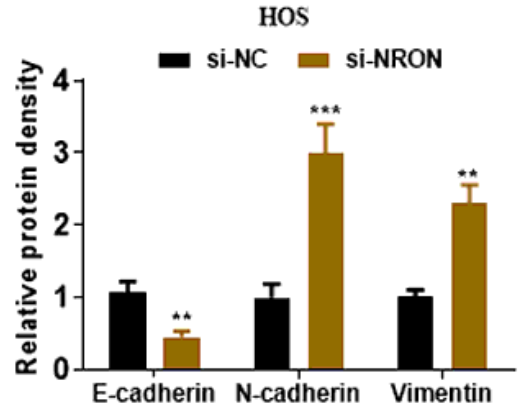

U20S

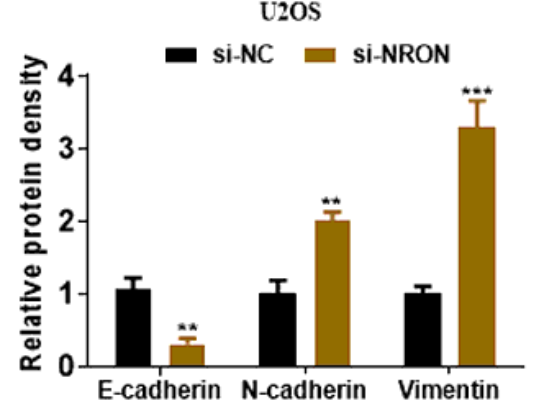

Figure 2

Knockdown of IncRNA NRON promoted cell proliferation. A. Knockdown efficiency was confirmed via realtime PCR. B. Knockdown of IncRNA NRON significantly promoted cell proliferation in HOS and U2OS cells. C. Knockdown of IncRNA NRON promoted cell invasion. D. Knockdown of IncRNA NRON decreased the protein level of E-cadherin, while increased the level of $\mathrm{N}$-cadherin and Vimentin. 


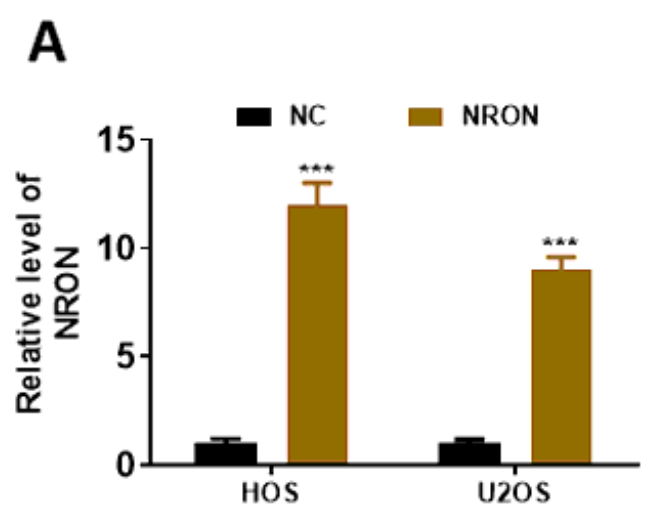

B
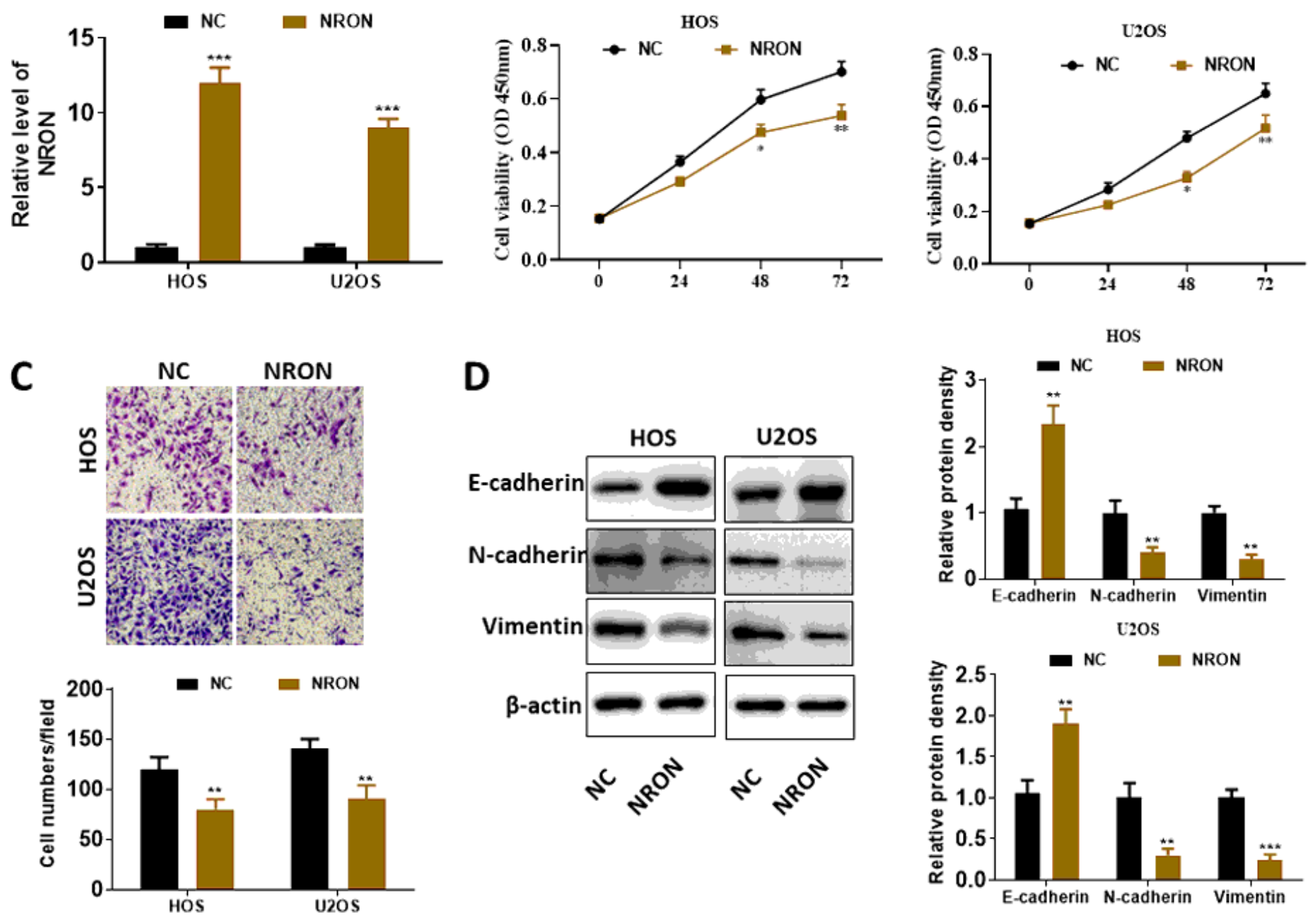

Figure 3

Overexpression of IncRNA NRON inhibited cell proliferation. A. Overexpression efficiency was confirmed via real-time PCR. B. Overexpression of IncRNA NRON inhibited cell proliferation. C. Overexpression of IncRNA NRON inhibited cell invasion. D. Overexpression of IncRNA NRON increased the protein level of Ecadherin and decreased the level of $\mathrm{N}$-cadherin and Vimentin. 
A

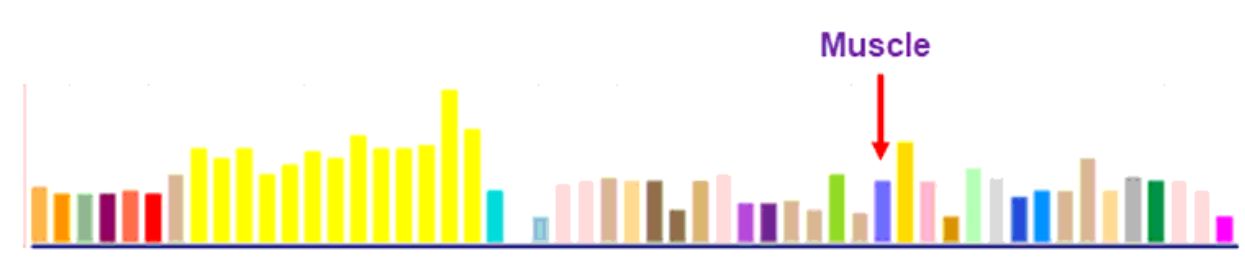

C

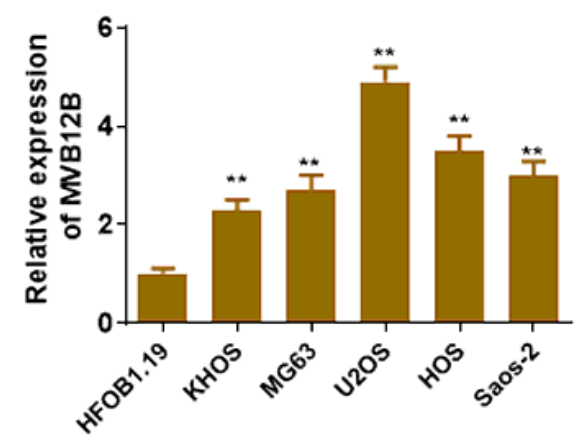

$\mathbf{F}$

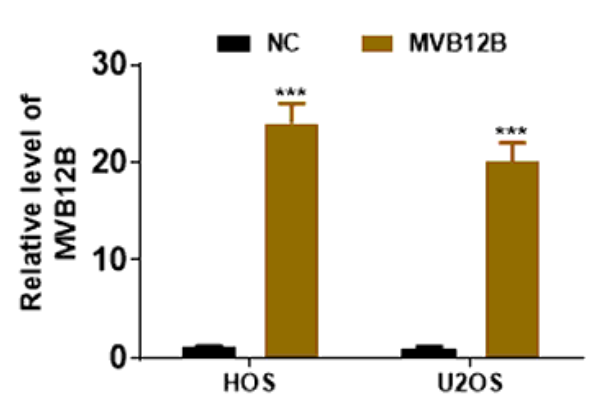

D

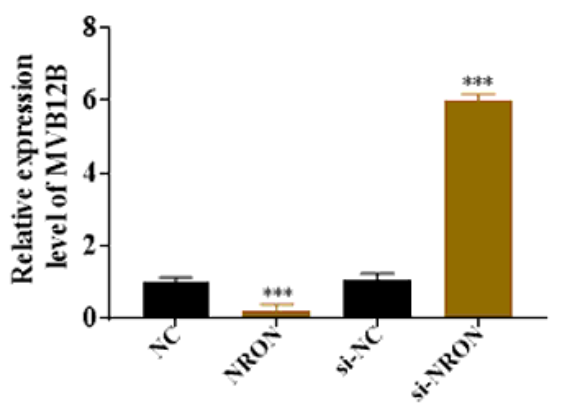

G

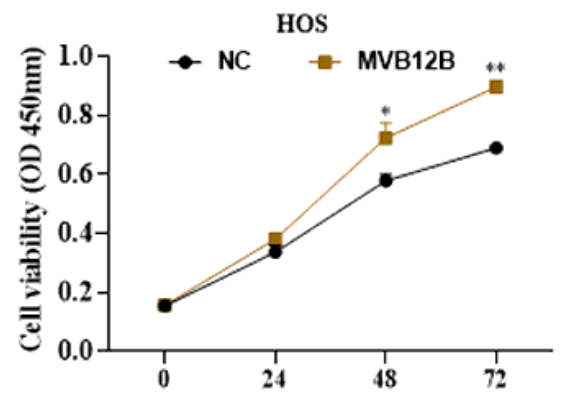

B

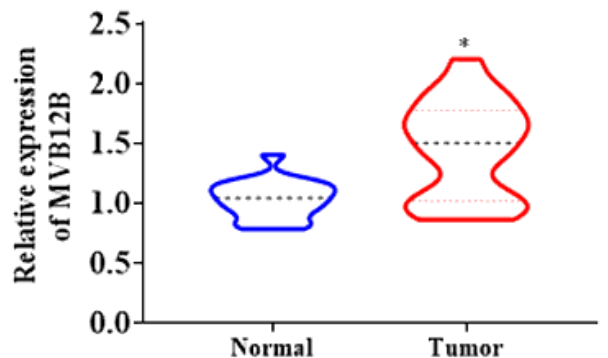

$\mathbf{E}$
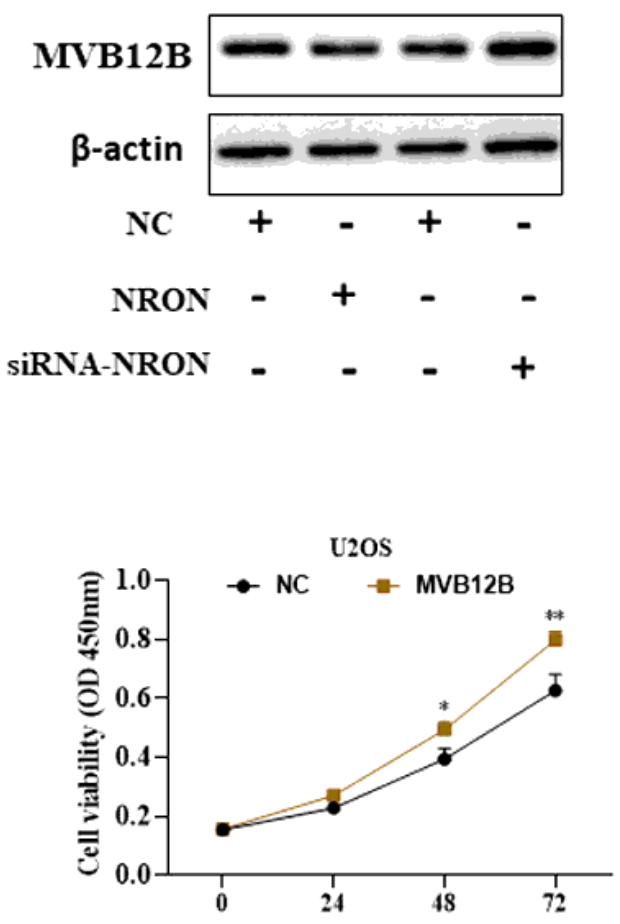

Figure 4

Biological features of MVB12B. A. Biological features of MVB12B in different tissues. B. The expression of MVB12B was significantly increased in tumor tissues. $C$. The expression of MVB12B was significantly increased in cell lines. D. IncRNA NRON negatively regulate the expression of MVB12B via real-time PCR. E. IncRNA NRON negatively regulate the protein level of MVB12B via western blot. F. Overexpression efficiency of MVB12B was confirmed via real-time PCR. G. Overexpression of MVB12B promoted cell proliferation in HOS and U2OS cells. 
A
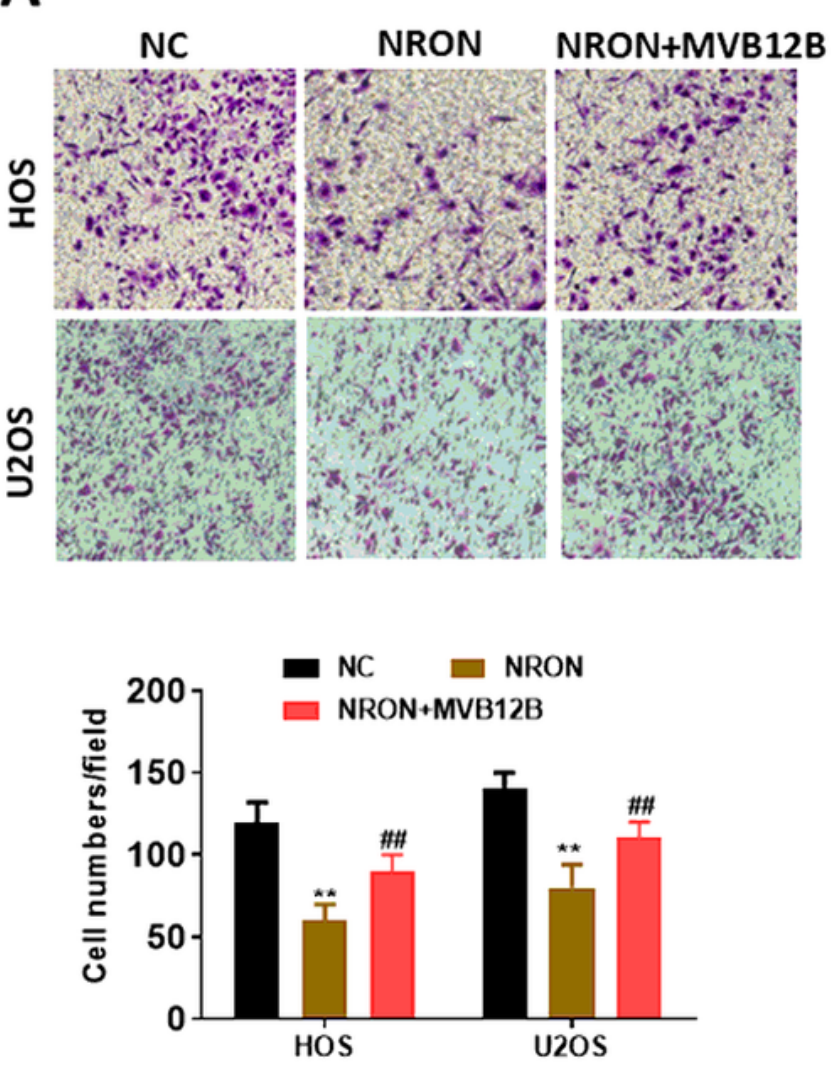

C

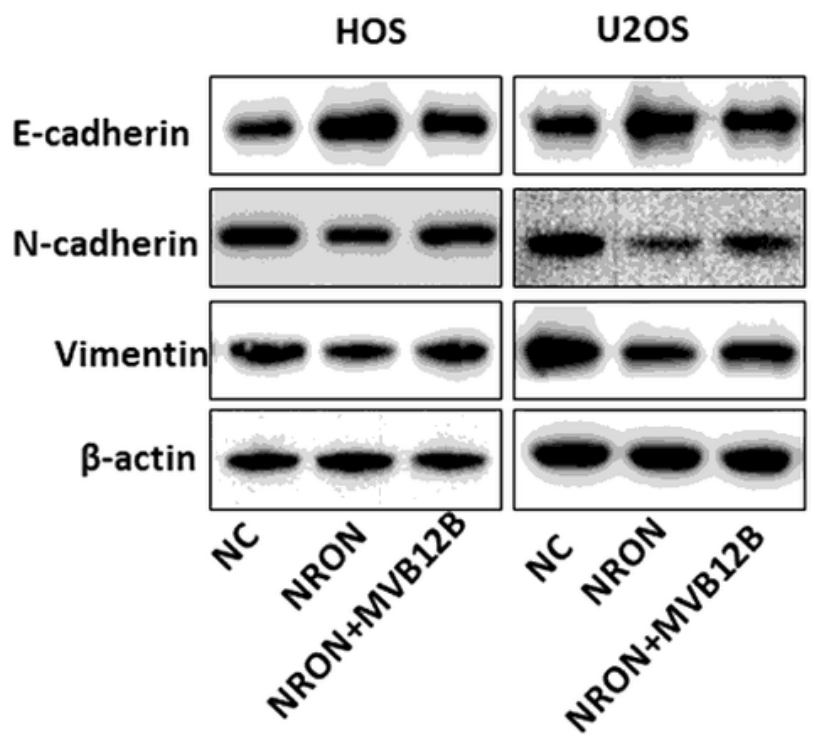

B
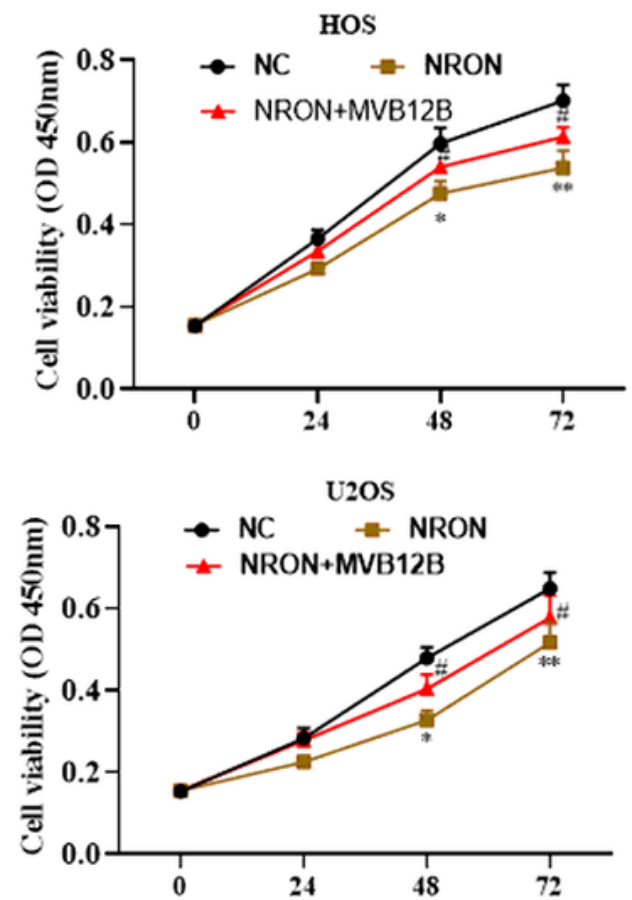

D

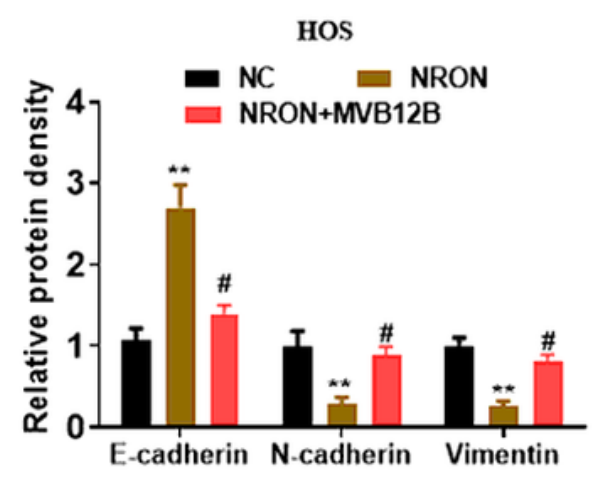

U2OS

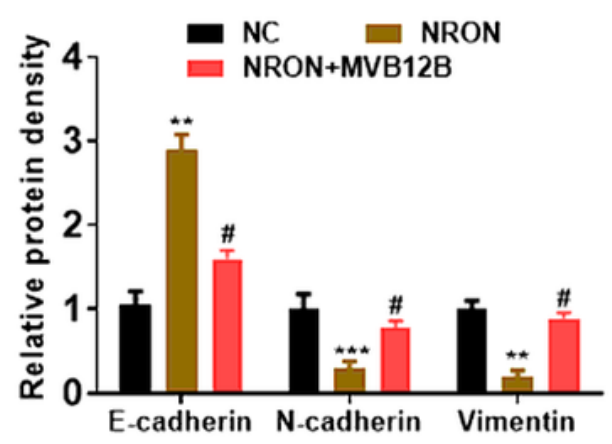

Figure 5

Reintroduction of MVB12B rescued the function of IncRNA NRON. A. Co-transfection of MVB12B and IncRNA NRON rescued the invasion effect of IncRNA NRON. B. Proliferation rates were measured by CCK8. C. Co-transfection of MVB12B and IncRNA NRON decreased the protein level of E-cadherin compared with IncRNA NRON group. D. Quantification data of western blot. 